\title{
The Role of a Self-Directed Technology to Improve Medication Adherence in Heart Failure Patients
}

\author{
Cynthia M. Walker \\ Faculty: Betty L. Elder \\ School of Nursing, College of Health Professions
}

\begin{abstract}
Medication nonadherence contributes to high morbidity and mortality in patients with heart failure. The purpose of this study was to investigate if a self-directed medical technology, MyMedSchedule.com ${ }^{\circledR}$, improved medication adherence. Thirty-three heart failure patients were recruited to participate in a pre-post-intervention. Morisky Medication Adherence Scale-8 item $($ indicated medication adherence was improved but not statistically significant, $\mathrm{z}=-1.661, \mathrm{p}=.097$ (pre-intervention scores $\mathrm{M}=6.44, \mathrm{SD}=1.38$; post intervention scores $\mathrm{M}=6.89, \mathrm{SD}=1.09)$. Participants expressed comfort (82\%), satisfaction (75\%), and confidence (75\%) using the technology. Technology-based strategies have potential to improve adherence and reduce poor outcomes.
\end{abstract}

\section{Introduction}

Heart failure (HF) is a costly complex chronic syndrome affecting millions in America. Affordability, age, patients' attitudes and beliefs, forgetfulness, and complex scheduled regimes are cited as barriers to medication adherence [1]. Medication is the cornerstone of the treatment of HF patients. Poor adherence to prescribed medications increases unnecessary hospitalizations, emergency room visits, and poor quality of life. Medication nonadherence contributes to high morbidity and mortality. MyMedSchedule.com ${ }^{\circledR}$, a free user-friendly web-based self-directed medication program, has been shown to improve medication adherence in patients with chronic diseases [2]. MyMedSchedule.com ${ }^{\circledR}$ gives patients and families the ability to maintain their medication schedules, set refill reminders, receive reminders to take their medications by text or email, and receive medication updates.

\section{Experiment, Results, Discussion, and Significance}

The current study is an exploratory pre-post-intervention design to investigate the use of a self-directed technology, MyMedScheule.com ${ }^{\circledR}$, to improve medication adherence of a purposively selected, non-random sample of 33 adult HF patients at a Heart Failure Disease Management Program (HF-DMP) in Kansas. All participants had a primary diagnosis of chronic HF documented by a physician, adequate proficiency at written and spoken English, and free of cognitive impairment. Following IRB approval from Wichita State University and Via Christi Health, the study was initiated. Informed and written consent were obtained. Human rights were protected by removing personal identifiers from the study questionnaires and replaced with a code number and all Protective Health Information secured in a locked cabinet at the clinical facility for at least 3 years.

Participants accessed MyMedSchedule.com ${ }^{\circledR}$ from a mobile application downloaded on a personal electronic device or from any electronic device; home computer, with internet capabilities. Training and technical support was provided at study enrollment, during HF-DMP visits, or by phone. Participants also received step-by-step written instructions. Staff training was conducted prior enrollment and weekly thereafter.

Participants completed the Morisky Medication Adherence Scale-8 item (MMAS-8) and HF Sociodemographic Questionnaire at baseline. The MMAS-8 and HF Technology Feasibility-Acceptance Questionnaire; explored attitudes and beliefs about using technology, were completed at the end of the project over the phone.

The Diffusion of Innovations (DOI) and Precaution Adoption Process Model (PAPM) [3] were utilized to provide a theoretical framework for this study. The primary concern of the DOI theory is the process by which a technology, MyMedSchedule.com ${ }^{\circledR}$, is exposed to and makes its way into the population and is (or is not) adopted. PAPM is a change behavior model with 5 stages: Unaware of the Issue, Unengaged by Issue, Deciding About Acting, Deciding Not to Act, Deciding to Act, Acting, and Maintenance. This model was used to identify and address changes in behaviors as the HF patient adapted to the use of the new technology. 
Descriptive statistics were used to examine participant's demographic characteristics. Inferential statistics, Wilcoxon Signed Rank Test, was used to examine the difference between the median pre-post MMAS-8 scores. The Spearman rho correlation coefficient was used to examine the relationship between participant's demographics (e.g., age, living status, medication frequency) and the MMAS-8 post intervention scores.

\section{Results}

Participants included 33 pre-MMAS-8 and 28 post MMAS-8 scores and HF Feasibility and Acceptance responses. Ages range from $28-85$ years with a mean age of 58.4 years ( $\mathrm{SD}=15.72$ years) and $27 \%$ over 70 years. Participants were majority male (72.7\%), living with someone (78\%), and HF Stage C with an ejection fraction less than $40 \%$ (72.7\%); poor heart function. All participants were on HF approved medications; beta blocker (100\%), angiotensin enzyme converting inhibitor or angiotensin receptor blocker (54\%). Over half (57.6\%) were on 11 or more medications and only $12 \%$ paying totally out-of pocket for medications. Weak correlations between participant's demographic variables and post-MMAS-8 intervention scores were not found significant.

Project Question 1: Does the use of a self-directed technology based intervention improve medication adherence in patients with heart failure? The results indicated the technology did not significantly improve medication adherence, difference between pre-post intervention scores, $\mathrm{z}=-1.661, \mathrm{p}=.097$ (pre-intervention scores $\mathrm{M}=6.44, \mathrm{SD}=1.38$; post- intervention scores $\mathrm{M}=6.89, \mathrm{SD}=1.09$ ). The mean rank for the MMAS-8 pre intervention score was 9.0 $($ median $=6.75)$ and post-intervention score $10.46($ median $=7.0)$.

Project Question 2: What is the feasibility and acceptance in using a technology-based intervention to improve medication adherence? Twenty-three (82\%) expressed comfort, $75 \%$ reported being satisfied and confident using the program. The technology was found not to be difficult to use by most participants (78.5\%). Overall, twenty-five participants reported making changes in how they took their medication after using MyMedSchedule.com®.

\section{Discussion}

A self-directed technology, MyMedSchedule.com ${ }^{\circledR}$, improved adherence to prescribed HF medication regimes of some participants. Affordability, age, and complexity of medication regime did not appear to be barriers to medication adherence in this sample. Participants reported positive responses after utilizing the medication adherence application. A high degree of acceptance and satisfaction was also found in those over 70 . This is consistent with the findings in the literature [4].

\section{Conclusions}

Medication nonadherence is complex and multidimensional. Poor adherence to prescribe HF medications leads to increased risk of morbidity, mortality, and enormous health care costs. Technology- based interventions such as MyMedSchedule.com ${ }^{\circledR}$ has the potential to improve medication adherence in patients with HF.

\section{References}

[1] Dunlay, S.M., Eveleth, J.M., Shah, N.D., McNallan, S.M., \& Roger, V.L. (2011). Medication adherence among community-dwelling patients with heart failure. Mayo Clinic Proceedings, 86(4), 273-281. doi: 10.4065/mcp.2010.0732.

[2] Zelikovsky, N. (2009). Evaluation of MedActionPlan website with adolescent transplant patients. Paper presented at the International Transplant Nurse Society Symposium, Montreal, Canada. Retrieved from http://www.medactionplan.com/medactionplan/studies_itns.asp.

[3] Edberg, M. (Ed.). (2007). Essentials of health behavior: Social and behavioral therory inpublic health. Mississauge, Ontario: Jones \& Bartlett Learning.

[4] McGillicuddy, J.W., Weiland, A.K., Frenzel, R.M., Mueller, M., Brunner-Jackson, B.M., Taber, D.J., ...Treiber, F.A. (2013). Patient attitudes toward mobile phone-based health monitoring: Questionnaire study among kidney transplant recipients. Journal of Medical Internet Research, 15(1), e6. 